\title{
Fish oil supplementation reverses the effect of cholesterol on apoptotic gene expression in smooth muscle cells
}

\author{
Sonia Perales ${ }^{\dagger}$, Ma José Alejandre ${ }^{\dagger}$, Rogelio Palomino Morales, Carolina Torres, Ana Linares ${ }^{*}$
}

\begin{abstract}
Background: Nutritional control of gene regulation guides the transformation of smooth muscle cells (SMC) into foam cells in atherosclerosis. Oxidative stress has been reported in areas of lipid accumulation, activating proliferation genes. Suppression of oxidative stress by antioxidant administration reduces this activation and the progression of lesions. We hypothesized that fish oil consumption may protect against atherosclerotic vascular disease. The study objective was to determine the effects of dietary cholesterol and fish-oil intake on the apoptotic pathways induced by 25 -hydroxycholesterol $(25-\mathrm{HC})$ in SMC cultures.

Methods: An in vivo/in vitro cell model was used, culturing SMC isolated from chicks exposed to an atherogenic cholesterol-rich diet with $5 \%$ of cholesterol (SMC-Ch) alone or followed by an anti-atherogenic fish oil-rich diet with $10 \%$ of menhaden oil (SMC-Ch-FO) and from chicks on standard diet (SMC-C). Cells were exposed to 25-HC, studying apoptosis levels by flow cytometry (Annexin V) and expressions of caspase-3, c-myc, and p53 genes by quantitative real-time reverse transcriptase-polymerase chain reaction. Results: Exposure to 25-HC produced apoptosis in all three SMC cultures, which was mediated by increases in caspase-3, c-myc, and p53 gene expression. Changes were more marked in SMC-Ch than in SMC-C, indicating that dietary cholesterol makes SMC more susceptible to 25-HC-mediated apoptosis. Expression of p53 gene was elevated in SMC-Ch-FO. This supports the proposition that endogenous levels of p53 protect SMC against apoptosis and possibly against the development of atherosclerosis. Fish oil attenuated the increase in c-myc levels observed in SMC-C and SMC-Ch, possibly through its influence on the expression of antioxidant genes.
\end{abstract}

Conclusion: Replacement of a cholesterol-rich diet with a fish oil-rich diet produces some reversal of the cholesterol-induced changes, increasing the resistance of SMC to apoptosis.

\section{Background}

Gene expression is influenced by dietary fat [1], gene transcription is regulated by dietary polyunsaturated fatty acids [2], and the nutritional control of gene regulation guides the transformation of SMC into foam cells in atherosclerosis [3]. The present study focused on the effect of cholesterol and fish oil intake on the expression of apoptosis-regulating caspase-3, c-myc and p53 genes.

In the $1970 \mathrm{~s}$, a very low incidence of death from ischemic heart disease was observed in Greenland

\footnotetext{
* Correspondence: analinar@ugr.es

† Contributed equally

Department of Biochemistry and Molecular Biology I, Faculty of Sciences, Campus Universitario de Fuentenueva Avenida Severo Ochoa s/n 18071 University of Granada, Spain
}

(c) 2010 Perales et al; licensee BioMed Central Ltd. This is an Open Access article distributed under the terms of the Creative Commons Attribution License (http://creativecommons.org/licenses/by/2.0), which permits unrestricted use, distribution, and reproduction in any medium, provided the original work is properly cited. dence from several research lines supports the hypothesis that fish consumption may protect against opment of atherosclerosis in rhesus monkeys, rabbits, and humans [5-7], the $\mathrm{n}-3$ fatty acids present in fish oil also have several biological effects that may be antiatherogenic. In the last decade evidence had been provided, that other fatty acids compounds are responsible for the positive effects of fish oil, e.g. furan FA (F-acids). $\mathrm{F}$-acids are generated in large amounts in algae, but they are also produced by plants and microorganisms. Fish and other marine organisms as well as mammals consume F-acids in their food and incorporate them into phospholipids and cholesterol esters. The atherosclerotic vascular disease. Fish oil inhibits devel- 
biogenetic precursor of the most abundant F-acid, F6, is linoleic acid. F-acids react readily with peroxyl radicals to generate dioxoenes. The radical-scavenging ability of F-acids may contribute to the protective properties of fish and fish oil diets against mortality from heart disease. [8]. However, the mechanism by which fish oil exert their effects is not fully understood. It has been proposed that some of these effects occur via plasma lipids [9]. It is well established that menhaden oil supplementation of the diet of chicks produced drastic reductions in plasma cholesterol, VLDL, and low- and high-density lipoprotein (LDL and HDL) fractions [9]. Supplementation with $10 \%$ menhaden oil was also reported [10] to drastically inhibit of 3-hydroxy-3methylglutaryl-CoA (HMG_CoA) reductase activity in neonatal chick liver. In another study, supplementation of neonate chick diet with $10 \%$ menhaden oil for 7 days resulted in a significant hypocholesterolemia and hypotriglyceridemia [11].

However, the effects of fish oil on the regulation of apoptosis are less well known [6]. It has been reported that free cholesterol loading of macrophages induces apoptosis in atherosclerosis [12,13] and that apoptosis decreases after lipid lowering [14]. Furthermore, smooth muscle cells (SMC) in atherosclerotic plaques can be lost via apoptosis [15], destabilizing plaque and increasing the risk of thrombosis. The decision phase of apoptosis involves the expression of specific proand anti-apoptotic genes. If the sum of signals gives apoptosis as a result, the entire protein machinery for destruction is unleashed. In SMC, apoptosis can be promoted by deregulation of the expression of c-myc or E2F [16], which in turn can be regulated by $\mathrm{p} 53$. The expression of p53 alone can promote apoptosis in SMC [17]. In atherosclerosis, SMC are highly susceptible to p53-mediated apoptosis [18].

25-hydroxycholesterol (25-HC) induced apoptosis in a leukemia model system [19], and 25-HC treatment repressed expression of the proto-oncogene c-myc, an early response gene [20,21] whose product, c-Myc, is critical for the control of cell proliferation and differentiation [22]. It has also been reported that oxLDL and $\alpha$-tocopherol may influence c-Myc activation in human coronary SMC [23]. Enhanced expression of c-Myc mRNA was observed in human SMC cultured from aortic plaques [24], carotid atherosclerotic lesions [25], and vein graft hyperplasia [26]. The tumor suppressor gene p53 encodes a transcription factor that activates genes involved in growth arrest (p21, GADD45) and apoptosis (Bax, Fas, p53-induced genes) [27]. p53 induces apoptosis by transcriptional activation of proapoptotic genes/ repression of anti-apoptotic genes and also via nontranscriptional mechanisms. The cellular response to p53 depends on the cell type and p53 expression level and on the presence of other apoptotic stimuli. Thus, low-level p53 expression often induces growth arrest, whereas apoptosis is only induced by higher expression levels. The induction of growth arrest may also reduce the sensitivity of cells to apoptosis [27]. In human atherosclerosis, p53 expression is negatively correlated with cell proliferation markers [28]. Endogenous p53 levels protect vascular SMC and stromal cells against apoptosis while promoting apoptosis in macrophages, and they protect against atherosclerosis development [29]. It was reported that p53 is highly expressed in atherosclerotic lesions and is involved in oxidized LDL-induced apoptosis [30].

Our group has developed an experimental culture model of SMC isolated from aorta of cholesterol-fed chicks (Ch-SMC) and control chicks on a standard diet (C-SMC) cultured under the same conditions. It has proven suitable for in vitro study of the transformation into foam cells of SMC induced in vivo by cholesterol diet, isolating the cells before plaque formation [31]. Previous results with this model showed marked differences in proliferation and synthesis of DNA, RNA and protein between Ch-SMC and C-SMC, and these cell lines differed in lipid (especially cholesterol) synthesis and in the effects of inhibition on the first step of cholesterol biosynthesis $[31,32]$. In another study, Ch-SMC and C-SMC cultures showed altered HMG-CoA reductase activity and gene expression at transcriptional level [33]. Hence, a high-cholesterol diet induced changes in the HMG-CoA reductase gene expression in aortic SMC of the chicks. The HMG-CoA reductase mRNA concentration in SMC cultures showed a marked rise after feeding that was not correlated with the fluctuation of activity observed during feeding of the cells for $72 \mathrm{~h}$ [34].

The hypothesis of this study was that vascular SMC apoptosis gene expression may be affected during early atherosclerosis in the in vivo/in vitro SMC culture model of chick experimental hypercholesterolemia and that fish oil supplementation after cholesterol dietary could change these results. We previously characterized 25OHC-induced SMC apoptosis by studying antiapoptotic and proapoptotic (bcl-2, bcl-xl and bax) gene expression [35]. The objective of the present study was to characterize 25-HC-induced SMC apoptosis by studying of caspase-3, c-myc, and p53 gene expression in SMC-C, SMC-Ch, and SMC-Ch-FO cultures. SMC-Ch-FO cultures were isolated from aorta of chicks fed with high-cholesterol diet for 10 days and then with standard diet supplemented with $10 \%$ fish oil for a further 10 days. The results showed that the effects of the cholesterol diet on the expression of these genes were reversed by dietary supplementation with fish oil. 


\section{Methods}

Animals

The protocol of this study was approved by the Animal Laboratory Service of the University of Granada (Spain), and the chicks received humane treatment according to the regulations for Animal Research of the European Union. Newborn White Leghorn male chicks (Gallus domesticus), supplied by the Animal Laboratory Service of the University of Granada, were kept in a chamber with a light cycle from 9 am to $9 \mathrm{pm}$ and controlled temperature of $29-31^{\circ} \mathrm{C}$ with food and water available ad libitum.

\section{Diet and treatment}

The diet was started at hatching and maintained until the chicks were killed at 20 days. Water was always available. None of the chicks died a natural death during the treatment or developed any disease. Three groups of 20-day-old chicks were used: control diet group (Cgroup), kept on a standard diet (Sanders A-00); Chtreatment group (Ch-group), fed on the same diet supplemented with $5 \% \mathrm{w} / \mathrm{w}$ powdered cholesterol mixed homogeneously (Panreac reagent Barcelona, pure grade); and a $\mathrm{Ch}$-fish oil group (Ch-FO-group), fed for 10 days with the $5 \%$ cholesterol-supplemented diet and then for a further 10 days with the standard diet supplemented with $10 \%$ fish (menhaden) oil. Experimental diets were prepared daily to minimize oxidation. The standard diet contained (w/w) 42\% carbohydrate (mainly starch), 3.5\% fat and $20.5 \%$ protein. The fatty acid composition of the diets is given in Table 1[11]. No significant differences in fatty acid composition were found among the diets. After the treatments, animals were anaesthetized with ketamine $(60 \mathrm{mg} / \mathrm{kg}$ of body weight) and sodium pentobarbital (50 mg/kg of body weight) before decapitation and removal of the aortic arch. No animals suffered at any stage of the experiment, and the study protocol was approved by the ethics committee of our university.

\section{Smooth muscle cell culture}

SMC were isolated from the aortic arch of the chicks as described elsewhere with slight modifications [31] and cultured in Dulbecco's Modification of Eagle's Medium (DMEM) supplemented with D-glucose (4.5 g/l), L-glutamate $(0.584 \mathrm{~g} / \mathrm{ml})$, antibiotic cocktail composed of penicillin $(100 \mathrm{mg} / \mathrm{ml})$ and amphotericin $(0.25 \mathrm{mg} / \mathrm{ml})$ (Sigma-Aldrich, Inc), and 10\% (v/v) fetal bovine serum (FBS). Medium was buffered with bicarbonate, and cultures were kept at $37^{\circ} \mathrm{C}$ in humidified atmosphere $(95 \%$ air, $5 \% \mathrm{CO}_{2}$ ). Media were renewed three times a week. Secondary cultures were initiated after either low or high passages using $0.05 \% / 0.02 \%$ Trypsin-EDTA solution. All experiments were conducted using 3 or
Table 1 Fatty acid composition of control and experimental diet

\begin{tabular}{|c|c|c|}
\hline Fatty acid & Control & $+10 \%$ fish Oil \\
\hline 14 & 0.8 & 6.6 \\
\hline 16 & 22.3 & 22.3 \\
\hline 18 & 8.6 & 5.8 \\
\hline Total sat. & 31.7 & 34.8 \\
\hline $16: 1 n-7$ & 3.3 & 9.2 \\
\hline $18: 1 n-9$ & 32.4 & 19.5 \\
\hline $20: 1$ n-9 & 0.0 & 0.4 \\
\hline Total MUFA & 35.7 & 29.1 \\
\hline $18: 2 n-6$ & 24.6 & 10.7 \\
\hline $20: 2 n-6$ & 2.5 & 2.4 \\
\hline $20: 3 n-6$ & 1.1 & 0.6 \\
\hline $20: 4 n-6$ & 1.6 & 1.4 \\
\hline Total n-6 & 29.8 & 15.1 \\
\hline $18: 3 n-3$ & 0.8 & 0.6 \\
\hline $20: 5 n-3$ & 0.0 & 12.0 \\
\hline $22: 5 n-3$ & 1.7 & 1.6 \\
\hline $22: 6 n-3$ & 0.0 & 6.8 \\
\hline Total n-3 & 2.5 & 21.0 \\
\hline Total PUFA & 32.3 & 36.1 \\
\hline Total unsat. & 68.0 & 65.2 \\
\hline Sat./unsat. & 0.47 & 0.53 \\
\hline Sat./PUFA & 0.98 & 0.96 \\
\hline $20: 5 / 22: 6$ & 0.00 & 1.76 \\
\hline$n-3 / n-6$ & 0.08 & 1.39 \\
\hline
\end{tabular}

Source: Castillo et all [11]

Results (\% fatty acid) are the means of 3 determinations.

5 passages. Cells were identified as vascular SMC by their hill-and-valley configuration at confluence and positive fluorescence staining for smooth muscle actin and myosin.

\section{5-hydroxycholesterol treatment and cytotoxicity assay}

Cytotoxicity was analyzed by using the MTT assay (3(4,5-dimethylthiazol-2-yl)-2,5-diphenyltetrazolium, bromide; Sigma). SMC were plated in 96-well plates at a density of 25000 cells/well. After adhering during overnight culture, cells were treated for 24 and $48 \mathrm{~h}$ with $25-\mathrm{HC}(5-40 \mu \mathrm{g} / \mathrm{ml})$ dissolved in absolute ethanol. The final concentration of ethanol in the culture medium never exceeded $0.8 \%$ and no effect on culture was observed at or below this concentration.

MTT was dissolved in DMEM at a concentration of 5 $\mathrm{mg} / \mathrm{ml}$. An amount of this solution equal to $10 \%$ of the culture medium volume was added to cell cultures. 
After $2 \mathrm{~h}$, cultures were removed from the incubator and washed with PBS. The formazan crystals were solubilized by adding $200 \mu \mathrm{l}$ of solubilization solution $(0.05$ $\mathrm{N} \mathrm{HCl}$ in isopropanol). Metabolic activity was quantified by subtracting light absorbance at $630 \mathrm{~nm}$ from absorbance at $570 \mathrm{~nm}$.

\section{Apoptosis Assays by Flow Cytometry}

Apoptotic cell death in SMC was assessed by flow cytometry (Annexin V-FLUOS Staining Kit, Roche). The culture medium of each plate (containing cells detached during the cell death process) was recovered in a tube, and the cells were suspended by a brief trypsinization (0.05\% with EDTA) and washed twice with cold PBS. Cells were then resuspended in $100 \mu \mathrm{L}$ of $1 \mathrm{x}$ binding buffer (10 mM Hepes, pH 7.4, $150 \mathrm{mM} \mathrm{NaCl}, 2.5 \mathrm{CaCl}_{2}$, $1 \mathrm{mM} \mathrm{MgCl}, 4 \% \mathrm{BSA}$ ) to a density of $1 \times 10^{6} \mathrm{cells} / \mathrm{ml}$ with annexin V-fluorescein isothiocyanate (FITC) $(0.5$ $\mu \mathrm{g} / \mathrm{ml})$. Cells were gently mixed and incubated for 15 min at room temperature in the dark. They were then re-suspended in $1 \mathrm{x}$ binding buffer, and propidium iodide (PI) $(0.6 \mu \mathrm{g} / \mathrm{mL})$ at $4^{\circ} \mathrm{C}$ was added to each tube before analysis. Fluorescence was induced with the $488-\mathrm{nm}$ argon laser and monitored at $512 \mathrm{~nm}$ (FL1) for the FITC signal and $620 \mathrm{~nm}$ (FL2) for IP fluorescence on FACS Vantage (Becton Dickinson Immunocytometry System, San José, CA.). The log of annexin V-FITC fluorescence was plotted against the $\log$ of IP fluorescence.

\section{Real-Time PCR Analysis}

Total RNA was isolated with Tri-Reagent/Trizol (Invitrogen, Ltd, UK). Single-stranded cDNA was synthesized from $4 \mu \mathrm{g}$ total RNA using an Oligo(dT) ${ }_{12-18}$ as primer and PowerScript ${ }^{\mathrm{m}}$ reverse transcriptase (Clontech Laboratories, Inc., CAL). Real-time PCR was performed with the Fast Start DNA Master SYBR Green I Kit (Roche) and Light Cycler system (Roche). For the Light Cycler reaction, a master mix of the following reaction components was prepared to the indicated final concentration: $12.6 \mu \mathrm{L} \mathrm{H} \mathrm{H}_{2} \mathrm{O}, 2.4 \mu \mathrm{MgCl}_{2}(4 \mathrm{mM}), 1 \mu \mathrm{L}$ forward primer $(0.5 \mu \mathrm{M}), 1 \mu \mathrm{L}$ reverse primer $(0.5 \mu \mathrm{M})$, and $2.0 \mu \mathrm{L}$ of the Fast Start DNA Master SYBR Green I mix (Roche). The primer sequences used in this study are listed in Table 2 and were optimized at an annealing temperature of $55^{\circ} \mathrm{C}$. The cDNA of the genes studied in the different samples (treated in vivo and in vitro) were diluted 1:100 and amplified to obtain the $C p$ value for each sample. Light Cycler products of the different gene expressions were analyzed by agarose gel electrophoresis, and a Light Cycler melting curve was constructed to test for a single product at the end of each PCR reaction. A mathematical model developed by Pfaffl [36] was used for the relative quantification of caspase 3, c-myc, and p53 mRNA expression in real-time PCR with respect to the reference $\beta$-actin gene transcript.

\section{Statistical analysis}

Results are expressed as mean \pm SEM. Data were analyzed by Student's $t$-test or, when the variances of two datasets significantly differed, by Welch's alternate $t$-test InStat statistical package was used for the data analyses. A $p$-value $\leq 0.05$ was considered significant.

\section{Results}

25-hydroxycholesterol induces apoptosis in the three cell cultures types

The percentage apoptosis was quantified in the three types of SMC cultures (C, Ch, and $\mathrm{Ch}-\mathrm{FO})$ after in vivo treatments (baseline) and after in vitro treatments. Figure 1 depicts the flow cytometry results and the percentage of annexin V-FITC-positive cells; the percentages in the upper right and lower left quadrants of the Figure represent the SMC in early and late apoptosis at baseline and after 24-h incubation with $20 \mu \mathrm{g} / \mathrm{ml} 25$-hydroxycholesterol, respectively. At baseline, the SMC showed a very low level of apoptosis (about $4 \%$ )

Cells showed a marked increase in apoptosis after the addition of $25-\mathrm{HC}(\mathrm{p}<0.001)$, with a higher increase in SMC-Ch than in SMC-C $(\mathrm{p}<0.01)$.

Expression of c-myc, p53, and caspase-3 genes in the cell culture model

At baseline, no significant difference in c-myc expression (Figure 2) was observed among SMC-C, SMC-Ch or SMC-Ch-FO. The expression of p53 gene (Figure 3) was significantly higher in SMC-Ch-FO than in the other two cultures $(p<0.001)$. None of the SMC

Table 2 Primer sequences and PCR product lengths for $\beta$-actin, caspase 3 c-myc and p53

\begin{tabular}{llll}
\hline Gene & Forward primer & Reverse primer & length \\
\hline$\alpha$-actin \\
$\alpha \beta$-actin & GCTCCGGCAATGTGCAA & AGGTTCATGAGGTAGT & 515 \\
c-myc & & & 476 \\
p53 & AGCGAACGAGTCTGAATCCAGC & AGTTCTCCTCCTCGATCTTG & 160 \\
caspase 3 & ATGTGCAACAGTTCCTGCAT & GAAATCCTGTCGAGTGGAGCAGG & 269 \\
$3-3$ & TTAGATTCTGGTATTGAAGC & & \\
\hline
\end{tabular}



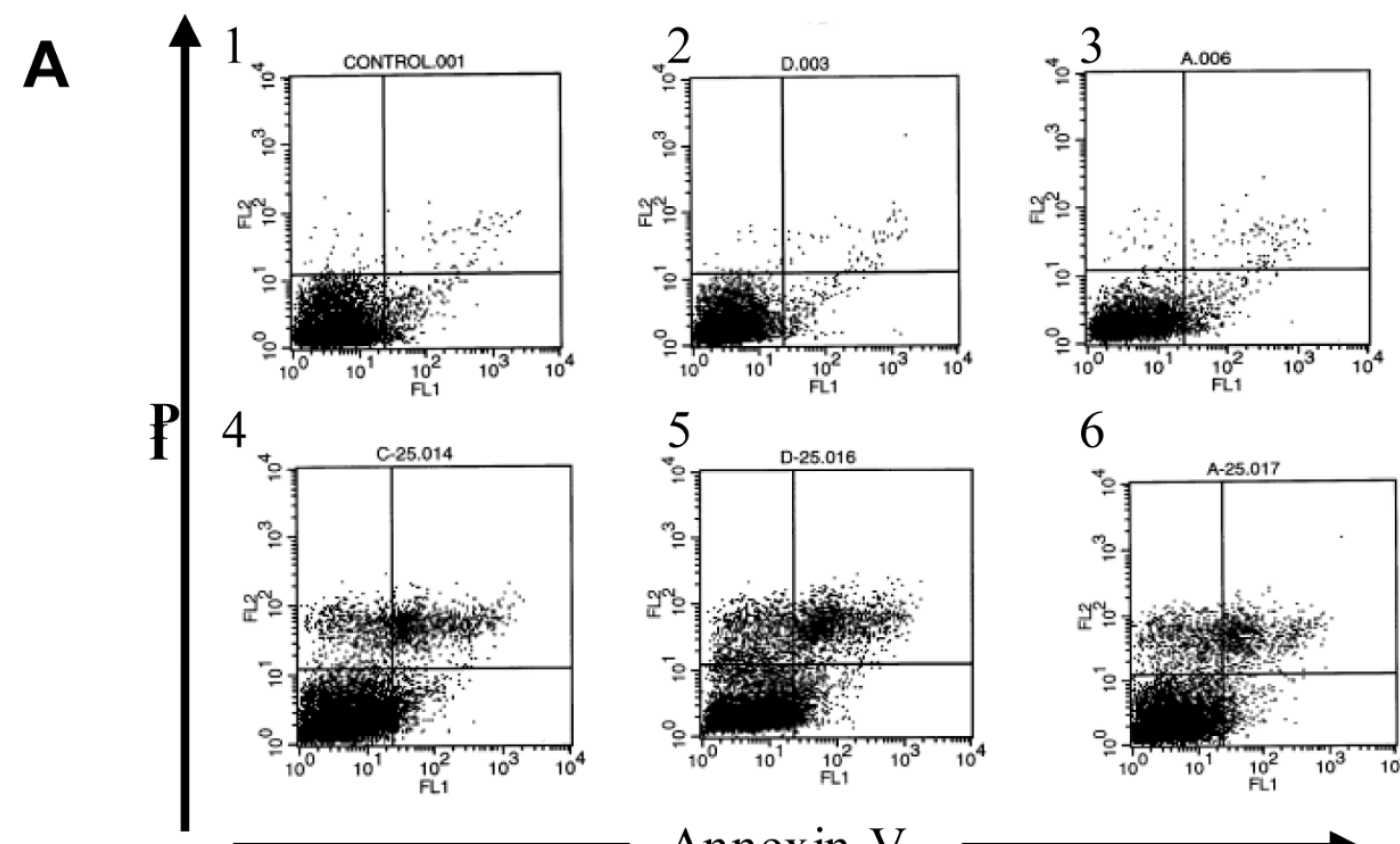

6

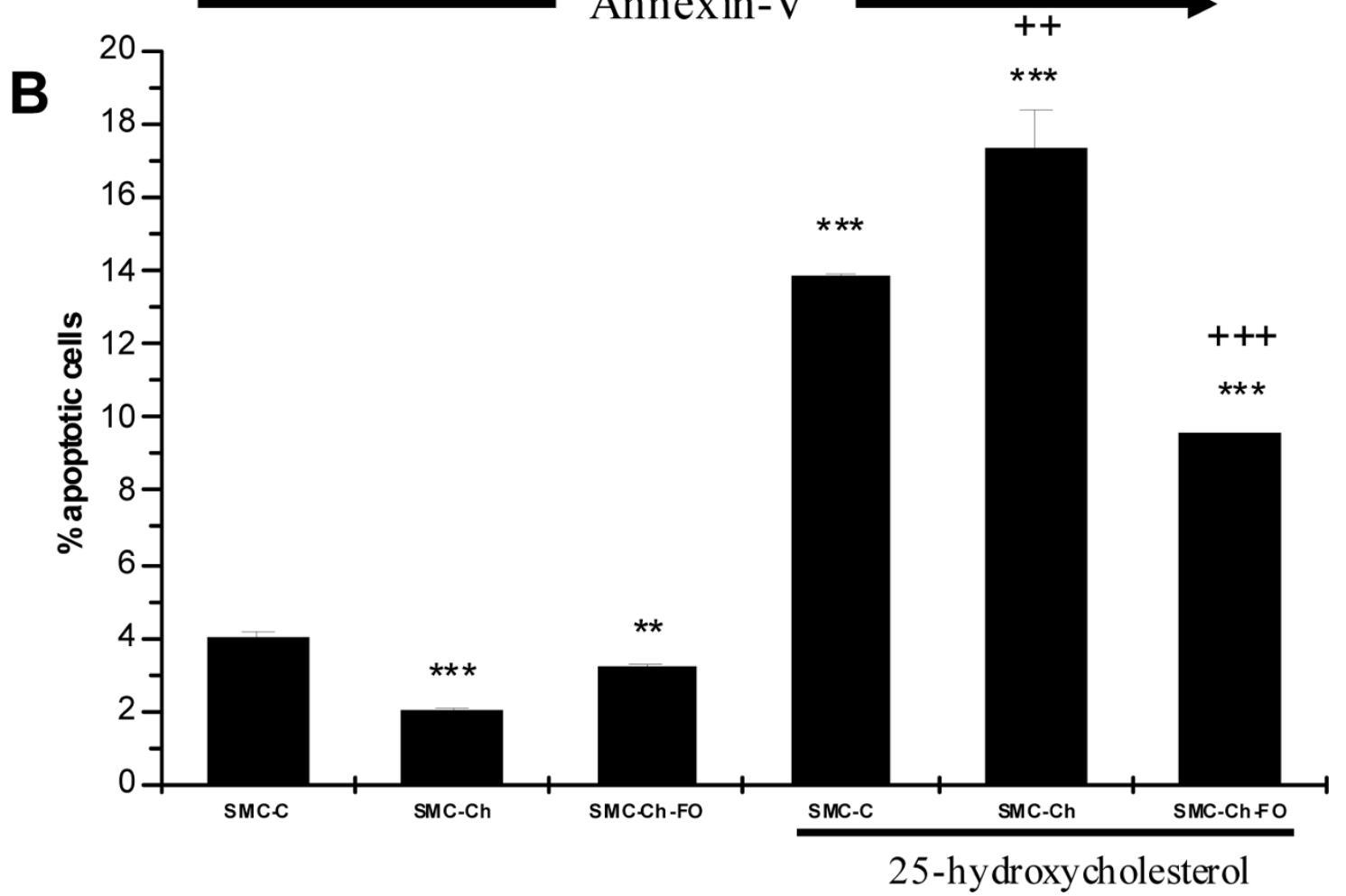

Figure 1 Apoptosis induced by 25 -Hidroxicholesterol in the cell culture model. Cells were labeled with Annexin V-FITC and PI (propidiun iodide), and apoptosis was assessed by flow cytometry. A, Representative scatter plots of PI (y-axis) versus Annexin V-FITC (x-axis). SMC-C (1), SMC-Ch (2), SMC-Ch-FO (3), SMC-C treated with $20 \mathrm{\mu g} / \mathrm{ml}$ 25-hydroxycholesterol (25-HC) for $24 \mathrm{~h}$ (4), SMC-Ch treated with $20 \mathrm{\mu g} / \mathrm{ml} 25-\mathrm{HC}$ for 24 h (5), and SMC-Ch-FO treated with $20 \mu \mathrm{g} / \mathrm{ml} 25-\mathrm{HC}$ for $24 \mathrm{~h}$ (6). Absence of both markers (lower left quadrants) indicates viable cells; PI positive alone (upper left quadrants) indicates cell necrosis, whereas Annexin V staining alone or together with PI (upper right and lower right quadrants) indicates of early- and late-stage apoptosis, respectively. B, Summary data of three experiments showing the \% of annexin- $V$ positive cells (upper right and lower right quadrants) in SMC cultures at baseline (SMC-C, SMC-Ch, and SMC-Ch-FO) and after 24-h treatment in vitro with $20 \mu \mathrm{g} / \mathrm{ml}$ $25-\mathrm{HC}$ for 24 h. ${ }^{*} P<0.05$, ${ }^{* *} P<0.01$, ${ }^{* * *} P<0.001$ vs. SMC-C, $+P<0.05,++P<0.01,+++P<0.001$ vs. SMC-C treated with $20 \mu \mathrm{g} / \mathrm{ml} 25-\mathrm{HC}$ for $24 \mathrm{~h}$. 


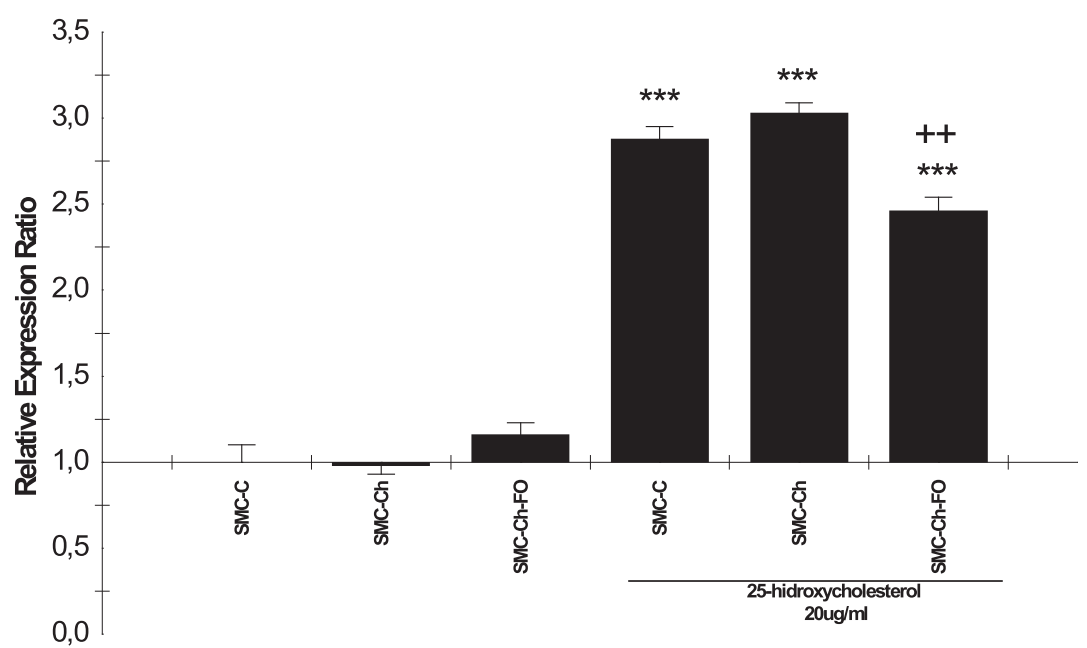

Figure 2 25-hidroxycholesterol effects on c-myc expression in the cell culture model. c-myc mRNA quantification in SMC cultures (SMC-C, SMC-Ch, SMC-Ch-FO) and SMC cultures treated with $20 \mu \mathrm{g} / \mathrm{ml}$ 25-hydroxycholesterol for $24 \mathrm{~h}$. mRNA levels were quantified by real-time semiquantitative reverse-transcription PCR. Results are shown as relative expression ratio of c-myc in SMC cultures with respect to control culture and expressed in comparison to reference gene $\beta$-actin. ${ }^{*} P<0.05$, ${ }^{* *} P<0.01,{ }^{* * *} P<0.001$ vs. SMC-C, $+P<0.05,++P<0.01,+++P<0.001$ vs. SMC-C treated with $20 \mu \mathrm{g} / \mathrm{mL}$ 25-hydroxycholesterol.

cultures showed elevated caspase- 3 expression (Figure $4)$, consistent with the baseline percentages of apoptosis (Figure 1).

\section{Effect of 25-hydroxycholesterol on the expression of c-myc, p53 and caspase-3 genes}

Addition of $25-\mathrm{HC}$ to the cultures produced a significant increase in $\mathrm{c}$-myc expression $(\mathrm{p}<0.001)$, with a significantly lower increase in SMC-Ch-FO versus SMC-C ( $\mathrm{p}<$
0.01) (Figure 2). Addition of 25-HC also produced a major increase in p53 mRNA ( $<<0.001)$ in all three culture types (Figure 3 ). Finally, $25-\mathrm{HC}$ produced a marked increase in caspase-3 gene expression (Figure 4).

\section{Discussion}

The dedifferentiation and proliferation/apoptosis of SMC in the arterial intima are among changes in early atherosclerotic lesions, when the disease is still

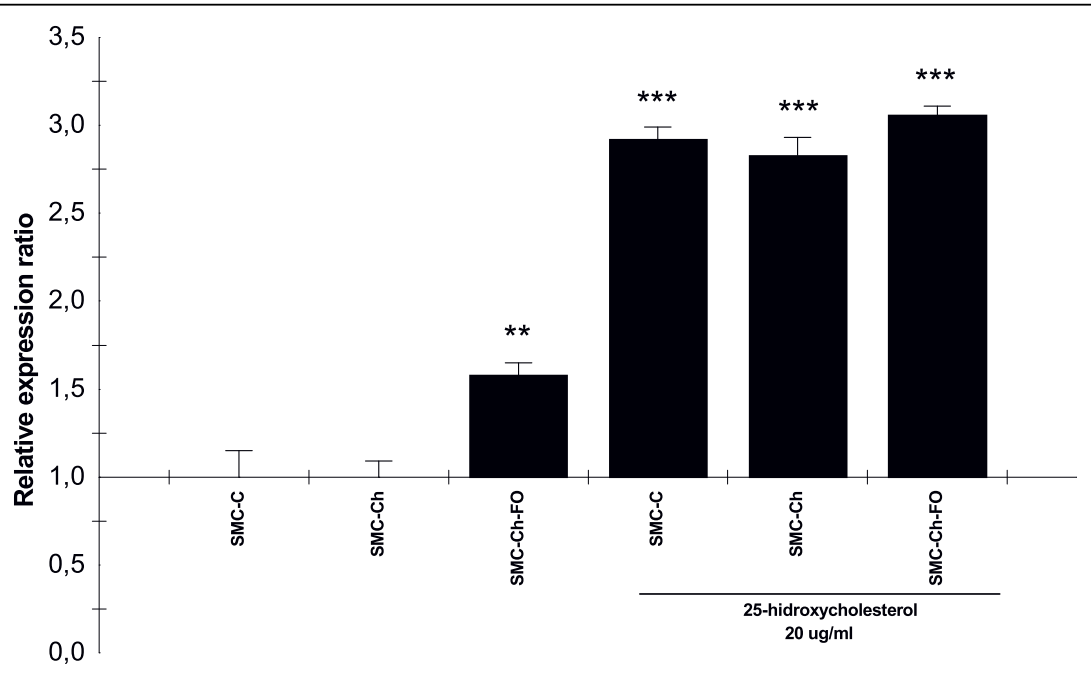

Figure 3 25-hidroxycholesterol effects on p53 expression in the cell culture model. p53 mRNA quantification in SMC cultures (SMC-C, SMC-Ch, SMC-Ch-FO) and SMC cultures treated with $20 \mu \mathrm{g} / \mathrm{mL}$ 25-hydroxycholesterol for $24 \mathrm{~h}$. mRNA levels were quantified by real-time semiquantitative reverse-transcription PCR. Results are shown as relative expression ratio of p53 in SMC cultures with respect to control culture and expressed in comparison to reference gene $\beta$-actin. ${ }^{*} P<0.05$, ${ }^{* *} P<0.01$, ${ }^{* *} P<0.001$ vs. SMC-C, $+P<0.05,++P<0.01,+++P<0.001$ vs. SMC-C treated with $20 \mu \mathrm{g} / \mathrm{mL}$ 25-hydroxycholesterol. 


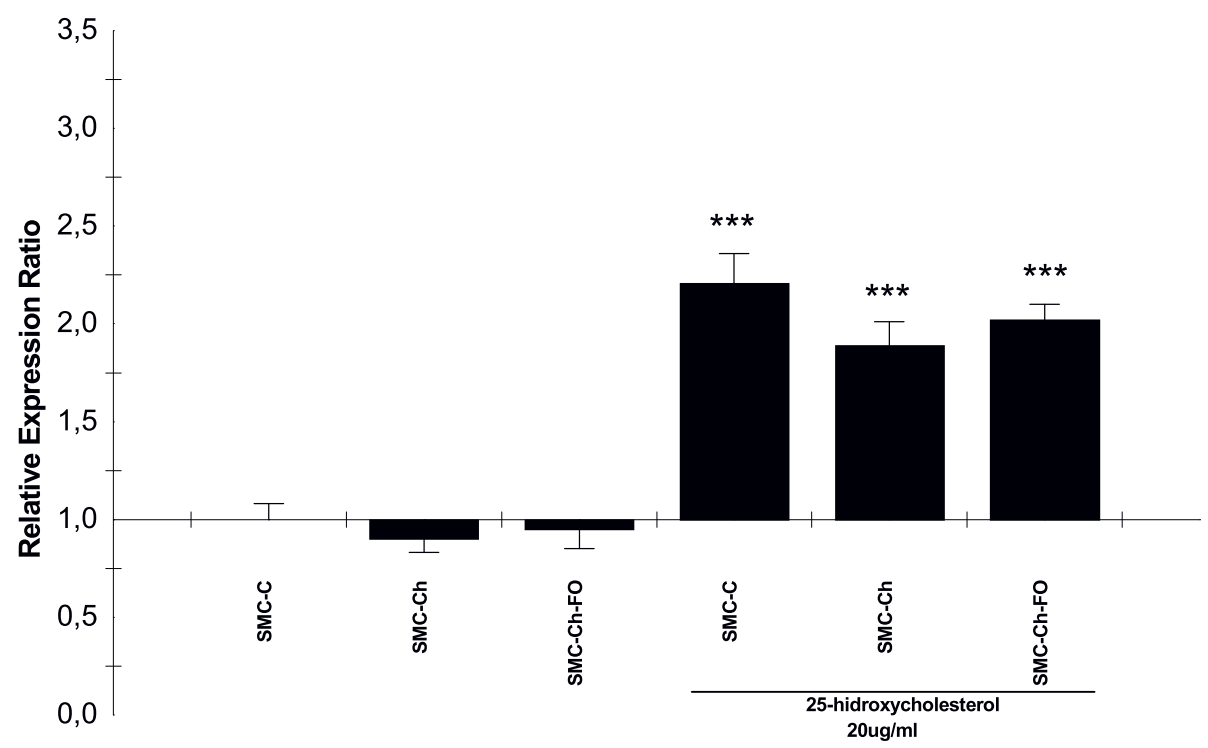

Figure 4 25-hidroxycholesterol effects on caspase-3 expresión in the cell culture model. caspase-3 mRNA quantification in SMC cultures (SMC-C, SMC-Ch, SMC-Ch-FO) and SMC cultures treated with $20 \mu \mathrm{g} / \mathrm{mL} 25$-hydroxycholesterol for $24 \mathrm{~h}$. mRNA levels were quantified by semiquantitative real-time reverse-transcription PCR. Results are shown as relative expression ratio of caspase-3 in SMC cultures with respect to control culture and expressed in comparison to reference gene $\beta$-actin. ${ }^{*} P<0.05,{ }^{*} P<0.01,{ }^{* * *} P<0.001$ vs. SMC-C, $+P<0.05,++P<0.01,++$ $+\mathrm{P}<0.001$ vs. SMC-C treated with $20 \mu \mathrm{g} / \mathrm{mL} 25$-hydroxycholesterol.

reversible [37,38]. The very low level of apoptosis shown in SMC in this study is similar to the $4 \%$ death rate usually observed in cultured cells. These data are in agreement with previous reports that cultured SMC from the arterial media, even those from atherosclerotic plaque, show no apoptosis in culture, unlike SMC from the intima [39]. In early atherosclerosis, cells in the arterial media that proliferate and migrate, modulating their phenotype, are protected against apoptosis in some manner [40].

SMC from chicks fed for 20 days with a cholesterolrich diet (SMC-Ch) were more susceptible to apoptosis than those from control chicks on a standard diet (SMC-C). This may be due to the accumulation of lipids in SMC, since lipid accumulation in early atherosclerosis has been associated with cell proliferation [41,42]. In contrast, SMC apoptosis levels were lower in chicks fed for 20 days with fish oil after 20 days on a standard diet (SMC-Ch-FO) than in the control chicks $(\mathrm{p}<0.01)$. This finding can be explained in part by a reversal of the process after the end of the high-cholesterol diet, as observed in animals with experimental atherosclerotic plaques [43], and in part by the protective effect of fish oil against 25-HC-mediated apoptosis, since levels were even lower than in the control SMC.

The expression of p53 gene was elevated in SMC-ChFO, supporting the proposition that endogenous levels of p53 protect SMC against apoptosis and possibly against the development of atherosclerosis [44]. The main controversy is whether endogenous p53 predominantly regulates proliferation or apoptosis in SMC. It has been proposed that p53 is responsible for the quiescent state of SMC in the arterial media. During the first changes in early atherosclerosis, SMC acquire the capacity to migrate from the media to the intima, which requires p53 expression inhibition that may be produced by mitogens in the vessels [45], whose signals produce a rapid degradation of endogenous p53 levels. The migration and proliferation of intima SMC commences at this time, and they do not re-express p53 when cultured. If this reduction in endogenous p53 does not occur, the SMC enter into apoptosis.

It has been reported that c-myc expression interferes with the control of SMC proliferation [46], and cultured $\mathrm{SMC}$ from atherosclerotic plaque have shown an overexpression of c-myc with respect to control SMC [47]. Elevated LDL levels have been implicated in the increased expression of c-myc in atherogenesis [48]. It should be noted that c-myc is expressed in cells that actively proliferate, and it is able to induce apoptosis in the absence of growth factors [49]. Our results are consistent with findings that oxidized LDLs (oxLDLs) produce an increase in c-myc expression [50]. Hence, dysregulation of c-myc causes SMC to proliferate but simultaneously reduces $\alpha$-actin and apoptosis levels, corresponding to a change from a contractile to synthetic SMC phenotype [46]. Our observation of lower c-myc levels in SMC-Ch-FO again indicates that 
these are protected against development of the disease. Oxysterols can produce oxidative stress in cells, thereby activating c-myc gene expression, which is sensitive to oxidative stress processes. Suppression of oxidative stress by the administration of anti-oxidants was found to attenuate this activation and development of the lesion [51]. Oxidative stress is created in areas of lipid accumulation, activating c-myc [51]. This study demonstrated that suppression of oxidative stress by antioxidant administration reduces this activation and progression of the lesion. Our findings indicate that fish oil can reduce the increase in c-myc levels observed in SMC-C and SMC-Ch, possibly through its influence on the expression of antioxidant genes [52].

The significant increase in p53 mRNA in the three culture types is consistent with findings that ox-LDLs (specifically 25-hydroxycholesterol) induce p53 [53]. A marked increase in Bax protein levels was also observed, possibly mediated by p53 [35]. These results indicate that, under our experimental conditions, 25-HC-induced apoptosis is mediated by p53. Finally, the marked increase in caspase- 3 gene expression induced by $25-\mathrm{HC}$ is in agreement with reports that oxysterol-induced apoptosis triggers the activation of caspase- 3 protein [54].

In conclusion, 25-hydroxycholesterol-induced apoptosis of SMC is mediated by a significant increase in cmyc and p53 levels. These changes were generally more marked in SMC-Ch than in SMC-C, indicating that dietary cholesterol produces changes in SMC that make them more susceptible to 25-hydroxycholesterolmediated apoptosis. Replacement of a cholesterol-rich diet with a fish oil-rich diet produces some reversal of the cholesterol-induced changes, increasing the resistance of SMC to apoptosis.

\section{Acknowledgements}

We thank Esther Marruecos for her technical support. This work was aided by grants from the Carlos III Institute of the Spanish Ministry of Health (EC08/00009).

\section{Authors' contributions}

All authors participated in the design of these studies and carried out the different assays. A.L. drafted the manuscript. All authors read and approved the final manuscript.

\section{Competing interests}

The authors declare that they have no competing interests.

Received: 9 June 2010 Accepted: 14 July 2010 Published: 14 July 2010

\section{References}

1. Jump DB, Clark SD: Regulation of gene expression by dietary fat. Ann Rev Ntr 1999, 19:63-90

2. Jump DB: Dietary polyunsaturated fatty acids and regulation of gene transcription. Curr Opin Lipidol 2002, 13:155-164.

3. Linares A, Perales S, Palomino-Morales RJ, Castillo M, Alejandre MJ: Nutricional control, Gene regulation and transformation of vascula smooth muscle cells in atherosclerosis. Cardiovasc Hematol Disord Drug Targets 2006, 6:151-168.

4. Bang HO, Dyeberg J, Hjorne N: The composition of food consumed by Greenland Eskimos. Acta Med Scand 1976, 200:69-73.

5. Nordoy A, Marchioli R, Arneses H, Videbaek J: n-3 Polyunsaturated fatty acids and cardiovascular diseases. Lipids 2001, 36:127-129.

6. Faggin E, Puato M, Chiavegato A, Franch R, Paulette P, Sartore S: Fish oil supplementation prevents neointima formation in nonhypercholesterolemic ballon-injured rabbit carotid artery by reducing medial and adventitial cell activation. Arterioscler Thromb Vasc Biol 2000, 20:152-163.

7. Chen MF, Hsu HC, Lee YT: Fish oil supplementation attenuates myointimal proliferation of the abdominal aorta after balloon injury in diet induced hypercholesterolemic rabbits. Prostaglandins 1995, 49:295-310.

8. Spiteller G: Furan fatty acids: occurrence, synthesis, and reactions. Are furan fatty acids responsible for the cardioprotective effects of a fish diet? Lipids 2005, 40(8):755-71.

9. Castillo M, Amalik F, Linares A, García-Peregrín E: Fish oil reduces cholesterol and arachidonic acid levels in plasma and lipoproteins from hypercholesterolemic chicks. Mol Cell Biochem 2000, 210:121-130.

10. Castillo M, Hortal JH, Gil-Vilariño A, Luque P, Iglesias J, García-Peregrín E: Differential effects of dietary fat on chick plasma and liver composition and HMG-CoA reductase activity. J Nutr Biochem 1999, 10:198-204.

11. Castillo M, Amalik F, Linares A, García-Peregrín E: Dietary fish oil reduces cholesterol and arachidonic levels in chick plasma and very low density lipoprotein. Mol Cell Biochem 1999, 200:59-67.

12. Yao PM, Tabas I: Free cholesterol loading of macrophages induces apoptosis involving the Fas Pathway. J Biol Chem 2000, 275:23807-23813.

13. Yao PM, Tabas I: Free cholesterol loading of macrophages is associated with widespread mitochondrial dysfunction of the mitochondrial dysfunction and activation of the mitochondrial apoptosis pathway. J Biol Chem 2001, 276:42468-42476.

14. Kockx MM, German AG: Apoptosis in atherosclerosis: beneficial or detrimental? Cardiovascular Res 2000, 45:736-746.

15. Kockx MM, De Meyer GRY, Muhring J: Apoptosis and related proteins in different stages of human atherosclerotic plaques. Circulation 1998, 97:2307-2315.

16. Bennett MR, Evan Gl, Newby AC: Deregulated c-myc oncogene expression blocks vascular smooth muscle cells inhibition mediated by heparin, interferon-, mitogen depletion and cyclin nucleotide analogues and induces apoptotic cell death. Circ Res 1994, 74:525-536.

17. Bennett MR, Evan Gl, Swartz SM: Apoptosis of rat vascular smooth muscle cells is regulated by p53 dependent and independent pathways. Circ Res 1995, 77:266-273.

18. Katayose D, Wersto R, Cowan K, Seth P: Consequences of p53 geneexpression by adenovirus vector on cell-cycle arrest and apoptosis in human aortic vascular smooth muscle cells. Biochem Biophys Res Commun 1995, 215:446-451.

19. Ayala-Torres S, Moller PC, Jhonson BH, Thompson EB: Characteristics of 25hydroxycholesterol-induced apoptosis in the human leukemic cell line CEM. Exp Cell Res 1997, 109:35-47.

20. Dang CV: c-Myc target genes involved in cell growth, apoptosis and metabolism. Mol Cell Biol 1999, 19:1-11.

21. Sakamuro D, Predergast GC: New C-Myc interacting proteins: a second myc network emerges. Oncogene 1999, 18:2942-2954.

22. Ayala-Torres S, Zhou F, Thompson EB: Apoptosis induced by oxysterol in CEM cells is associated with negative regulation of c-Myc. Exp Cell Res 1999, 246:193-202

23. Nigris F, Youssef T, Ciafre SA, Franconi F, Anania V, Condorelli GL, Palinski W, Napoli C: Evidence for oxidative activation of c-Myc-dependent nuclear signaling in human coronary smooth muscle cells an in early lesions of watanable heritable hyperlipidemic rabbits. Circulation 2000, 102:2111-2117.

24. Parkes JL, Cardell RR, Hubbard FC Jr, Hubbard D, Meltzer A, Penn A: Cultured human atherosclerotic plaque smooth muscle cells retain transforming potential and display enhanced expression of the myc protooncogene. Am J Pathol 1991, 138:765-775.

25. Marin ML, Gordon RE, Veith FJ, Tulchin N, Panetta TF: Distribution of c-myc oncoprotein in healthy and atherosclerotic human carotid arteries. $J$ Vasc Surg 1993, 18:170-177. 
26. Ramirez JA, Sanchez LA, Marin ML, Lyon RT, Parsons RE, Suggs WD, Veith FJ: c-Myc oncoprotein production in experimental vein graft intimal hyperplasia. J Surg Res 1996, 61:323-329.

27. Vousden $\mathrm{K}, \mathrm{Lu} \mathrm{X}$ : Live or let die: the cells response to $\mathrm{p} 53$. Nature reviewsCancer 2002, 2:594-604

28. Ihling C, Menzel G, Wellens E, Monting JS, Shaefer HE, Zeiher AM: Topographical association between the cyclin-dependent kinases inhibitor P21, p53 accumulation, and cellular proliferation in human atherosclerosis tissue. Art Thromb Vasc Biol 1997, 17:2218-2224.

29. Mercer J, Figg N, Stoneman V, Braganza D, Bennett MR: Endogenous p53 protects vascular smooth muscle cells from apoptosis and reduces atherosclerosis in apoE knockout mice. Circ Res 2005, 96:667-674.

30. Metzler $B, H u Y$, Dietrich $H, X u$ Q: Increased expression and activation of stress-activated protein kinases/c-Jun $\mathrm{NH}_{2}$-terminal protein kinases in atherosclerotic lesions coincide with p53. Am J Pathol 2000, 156:1875-1886.

31. Carazo A, Alejandre MJ, Diaz R, Rios A, Castillo M, Linares A: Changes in cultured arterial smooth muscle cells isolated from chicks upon cholesterol feeding. Lipids 1998, 33:181-190.

32. Carazo A, Alejandre MJ, Louktibi A, Linares A: The reversal of the inhibition on lipids synthesis by L-659,699 in arterial smooth muscle cells cultures. Mol Cell Biochem 2001, 221:25-31.

33. Carazo A, Alejandre MJ, Suarez MD, Linares A: Alterations in 3-hydroxy-3methylglutaryl-CoA reductase mRNA concentration in cultured chick aortic smooth muscle cells. Lipids 2000, 35:587-593.

34. Alejandre MJ, Perales S, Carazo A, Palomino-Morales R, Linares A: Cyclic fluctuations of 3-hydroxy-3-methylglutaryl-CoA reductase in aortic smooth muscle cell cultures. Lipids 2006, 41:1089-1099.

35. Sonia Perales, Ma José Alejandre, Rogelio Jesús Palomino-Morales, Carolina Torres, Jose Iglesias, Ana Linares : Effect of oxysterol-induced apoptosis of vascular smooth muscle cells on experimental hypercholesterolemia. Journal of Biomedicine and Biothecnology 2009, 2009:456208, Epub 2009 Aug 27.

36. Pfaffl MW: A new mathematical model for relative quantification in realtime RT-PCR. Nucleic Acid Res 2001, 1;29(9):e45.

37. Ross R: The pathogenesis of atherosclerosis: a perspective for the 1990s. N Engl J Med 1993, 362:801-809.

38. Campbell GR, Campbell JH: The role of smooth muscle cells in atherosclerosis. Curr Opin Lipidol 1994, 5:323-3303.

39. Bennett MR, Evan Gl, Swartz SM: Apoptosis of human vascular smooth muscle cells derived from normal vessels and coronary atherosclerotic plaques. J Clin Invest 1995, 95:2266-2274.

40. Mayr M, Xu Q: Smooth muscle cells apoptosis in arteriosclerosis. Exp Geron 2001, 36:969-987.

41. Kockx MM, De MG, Muhring J, Bult H, Bultinck J: Herman. Distribution of cell replication and apoptosis in atherosclerotic plaques of cholesterolfed rabbits. Atherosclerosis 1996, 120:115-124.

42. Hasdai D, Snangiorgi G, Spagnoli L, Simari RD, Holmes DR Jr, Kwon HM, Carlson PJ, Schawrtz RS, Lerman A: Coronary artery apoptosis in experimental hypercholesterolemia. Atherosclerosis 1999, 142:317-325.

43. Kockz MM, Herman AG: Apoptosis in atherosclerosis: beneficial or detrimental? Cardiovascular Res 2000, 45:736-746.

44. Mercer J, Figg N, Stoneman V, Braganza D, Bennett MR: Endogenous p53 protects vascular smooth muscle cells from apoptosis and reduces atherosclerosis in ApoE knockout mice. Circ Res 2005, 96(6):667-674.

45. Rodriguez-Campos A, Ruiz-Enriquez P, Faraudo S, Badimon L: Mitogeninduced p53 down-regulation precedes vascular smooth muscle cell migration from healthy tunica media proliferation. Arterioscler Thromb Vasc Biol 2001, 21:214-219.

46. Y Shi, Hutchinson HG, Hall DJ, Zalewski A: Downregulation of c-myc expression by antisense oligonucleotides inhibits proliferation of human smooth muscle cells. Circulation 1993, 88:1190 -1195.

47. Parkes JL, Cardell RR, Hubbard FC Jr, Hubbard D, Meltzer A, Penn A: Cultured human atherosclerotic plaque smooth muscle cells retain transforming potential and display enhanced expression of the myc protooncogene. Am J Pathol 1999, 138:765-775.

48. De Nigris F, Youssef T, Ciafré SA, Franconi F, Anania V, Condorelli GL, Palinski W, Napoli C: Evidence for oxidative activation of c-Myc dependent nuclear signaling in human coronary smooth muscle cells and in early lesions of Watanabe Heritable Hyperlipidemic Rabbits. Circulation 2001, 02:2111-2117.
49. Petengaris $S$, Khan M: The many faces of c-myc. Archives of biochemistry and biophysics. 2003, 416:129-136.

50. de Nigris F, Youssef T, Ciafre S, Franconi F, Anania V, Condorelli G, Palinski W, Napoli C: Evidence for oxidative activation of c-Mycdependent nuclear signaling in human coronary smooth muscle cells and in early lesions of Watanabe heritable hyperlipidemic rabbits: protective effects of vitamin E. Circulation 2000, 102:2111-2117.

51. Hajra L, Evans Al, Chen M, Hyduk SJ, Collins T, Cybulsky MI: The NF-kappaB signal transduction pathway in aortic endothelial cells is primed for activation in regions predisposed to atherosclerotic lesion formation. Proc Natl Acad Sci 2000, 97:9052-9057.

52. Takahashi M, Tsuboyama-Kasaoka N, Nakatani T, Ishii M, Tsutsumi S, Aburatani $\mathrm{H}$, Ezaki O: Fish oil feeding alters liver gene expressions to defend against PPARalpha activation and ROS production. Am J Physiol Gastrointest Liver Physiol 2002, 282:G338-G348.

53. Lee T, Chau L: Fas/Fas ligand-mediated death pathway is involved in oxLDL-induced apoptosis in vascular smooth muscle cells. Am J Physiol Cell Physiol 2001, 280:709-718.

54. Nishio E, Watanabe Y: Oxysterols induced apoptosis in cultured smooth muscle cells through CPP32 protease activation and bcl-2 protein downregulation. Biochim Biophy Res Commun 1996, 226:928-934.

doi:10.1186/1476-511X-9-70

Cite this article as: Perales et al:: Fish oil supplementation reverses the effect of cholesterol on apoptotic gene expression in smooth muscle cells. Lipids in Health and Disease 2010 9:70.

\section{Submit your next manuscript to BioMed Central and take full advantage of:}

- Convenient online submission

- Thorough peer review

- No space constraints or color figure charges

- Immediate publication on acceptance

- Inclusion in PubMed, CAS, Scopus and Google Scholar

- Research which is freely available for redistribution

Submit your manuscript at www.biomedcentral.com/submit
C Biomed Central 